\title{
Efecto a largo plazo de la infección por SARS-CoV-2:Síndrome neurológico post-Covid-19
}

\section{Long-term effect of SARS-CoV-2 infection: Post-Covid-19 neurological syndrome}

$\mathrm{DOI}$

https://doi.org/10.35434/rcmhnaaa.2021.143.1284

\section{RESUMEN}

Introducción: La pandemia de la COVID-19 ha provocado aproximadamente 182 millones de casos y cerca de cuatro millones de muertes en todo el mundo, comprometiendo con mayor frecuencia a personas de 25 y 64 años de edad. Esta infección afecta principalmente al sistema respiratorio, pero otros órganos y sistemas pueden verse afectados a corto plazo como el neurológico. Una gran población de pacientes ha logrado superar la infección por el SARS-CoV-2, sin embargo, han comenzado a reportarse una serie de manifestaciones clínicas tardías, básicamente neuropsiquiátricas, dentro de ellas se ha referido a la ansiedad, depresión, deterioro del sueño, dolor muscular, mareos, cefaleas, fatiga, anosmia, entre otros; a este conjunto de manifestaciones tardías se le ha denominado síndrome neurológico post-Covid-19 y requiere por un lado, la atención de la comunidad médica para investigar las manifestaciones tardías o secuelas de esta enfermedad y por otro lado, una vigilancia médica ante la consulta de pacientes con estas manifestaciones.

Palabras Clave: Covid-19; SARS-CoV-2; Síndrome neurológico postCOVID-19; desórdenes neurológicos (Fuente: DeCS-BIREME).

\section{ABSTRACT}

Background: The COVID-19 pandemic has caused approximately 182 million cases and nearly four million deaths worldwide, most frequently involving people between 25 and 64 years of age. This infection primarily affects the respiratory system, but other organs and systems can be affected in the short term, such as the neurological system. A large population of patients has managed to overcome the SARS-CoV-2 infection, however, a serie of late clinical manifestations have begun to be reported, basically neuropsychiatric, including anxiety, depression, disorder of sleep, muscle pain, dizziness, headaches, fatigue, anosmia, among others; this set of late manifestations has been called post-Covid-19 neurological syndrome and requires, on the one hand, the attention of the medical community to investigate the late manifestations or sequel of this disease and, on the other hand, medical vigilance when consulting patients with these manifestations.

Keywords: Covid-19; SARS-CoV-2; Post Covid-19 Neurological Syndrome; neurological disorders. (Source: DeCS-BIREME).

\section{FILIACIÓN}

1. Escuela de Medicina, Universidad Privada Antenor Orrego, Trujillo, Perú.

2. Hospital Regional Docente de Trujillo, Trujillo, Perú.

a. Estudiante de Medicina.

b. Doctor en Investigación Clínica y Traslacional

\section{ORCID}

1. Caballero-Alvarado José / 0000-0001-8297-6901

2. Camacho Vargas Edith / 0000-0003-0843-4834

3. Rojas Sánchez Pedro / 0000-0002-4225-7467

\section{CORRESPONDENCIA}

José Caballero Alvarado

Av. América Sur 3145, urb. Monserrate, Trujillo, Perú

Teléfono: (044) 604444 anexo $1000-1001$

EMAIL

jcaballeroalvarado@icloud.com

\section{CONFLICTOS DE INTERÉS}

Los autores niegan conflictos de interés.

\section{FINANCIAMIENTO}

Autofinanciamiento.

REVISIÓN DE PARES

Recibido: 02/07/2021

Aceptado: 20/09/2021

\section{COMO CITAR}

Caballero-Alvarado, J., Camacho-Vargas, E., \& RojasSánchez, P. Efecto a largo plazo de la infección por SARSCoV-2: Síndrome neurológico post-Covid-19. Revista Del Cuerpo Médico Hospital Nacional Almanzor Aguinaga A s e n jo, 2021,14 ( 3 ), $404-409$. https://doi.org/10.35434/rcmhnaaa.2021.143.1284 


\section{INTRODUCCIÓN}

A finales de 2019, aparecieron de manera repentina varios casos de neumonía atípica en la ciudad de Wuhan, China; cuatro semanas después la OMS declaró a esta nueva enfermedad como emergencia de salud pública de interés internacional. El 11 de febrero de 2020, la OMS denominó oficialmente dicha enfermedad como Enfermedad por Coronavirus-2019 (COVID-19) y el Comité Internacional de Taxonomía de Virus denominó al virus como SARS-CoV-2, siendo un miembro de la familia de los coronavirus que tienen una gran capacidad para infectar a los seres humanos ${ }^{(1)}$.

Diferentes recomendaciones de salud pública y tratamientos fueron implementados con la finalidad de reducir el contagio y, por ende, su expansión, mientras se lograban obtener las vacunas; las cuales representan la medida de salud pública más importante para proteger a la población de la COVID-19 en todo el mundo, dado que el SARS-CoV-2 es muy contagioso e infecta a poblaciones de forma amplia y global. Diferentes grupos de investigación de todo el mundo trabajaron contra carrera para obtener las vacunas y algunos de ellos lo lograron, actualmente diferentes países han iniciado la vacunación, protegiendo en primer lugar a su población vulnerable y otros de manera generalizada ${ }^{(2)}$.

Muchos pacientes que fueron infectados por el SARS-CoV-2 han logrado superar la enfermedad, sus complicaciones agudas y mediatas, sin embargo, semanas o meses después de la infección índice, se han comenzado a reportar síntomas tardíos en estos pacientes, incluso en aquellos que cursaron de manera leve o moderada; los síntomas frecuentes referidos por algunos pacientes son cefalea, fatiga, mialgias, mareos, anosmia, entre otros; a este conjunto de síntomas se ha denominado síndrome neurológico post COVID-19, algunos autores han comenzado a describirlos como una sintomatología similar a la presentada en la encefalomielitis miálgica (EM) / síndrome de fatiga crónica (SFC), fatiga persistente, mialgia difusa, síntomas depresivos y sueño no reparador $^{(3)}$.

La presente revisión propone realizar una exploración bibliográfica con la finalidad de ahondar en las manifestaciones neurológicas tardías post COVID-19.

\section{ESTRATEGIADE BÚSQUEDA}

Realizamos una búsqueda bibliográfica en PubMed, Scopus y Web of Science, en idioma inglés. La expresión de búsqueda utilizada fue la siguiente: (("Post- COVID 19 Neurological Syndrome" OR "Post Covid-19 Neurological Syndrome" OR "chronic fatigue syndrome" OR "Post-COVID-19 Syndrome" OR "New Persistent Daily Headache" OR "neuropsychiatric syndromes" OR “Long-Term Brain Disorders") AND ("COVID19" OR "SARS-CoV-2")). Se obtuvieron un total de 713 artículos, procedentes de las bases mencionadas y 7 procedentes de otras fuentes, todos ellos básicamente estudios observacionales, los 720 artículos fueron exportados a la plataforma Rayyan, en la que se seleccionaron las de mayor relevancia y novedad para el objetivo planteado, fueron descartados 460 artículos cuyos diseños no estuvieron acordes al objetivo. Finalmente, quedaron 20 artículos que constituyeron la bibliografía asociada al síndrome neurológico popst-COVID-19 de la presente investigación (Figura 1).

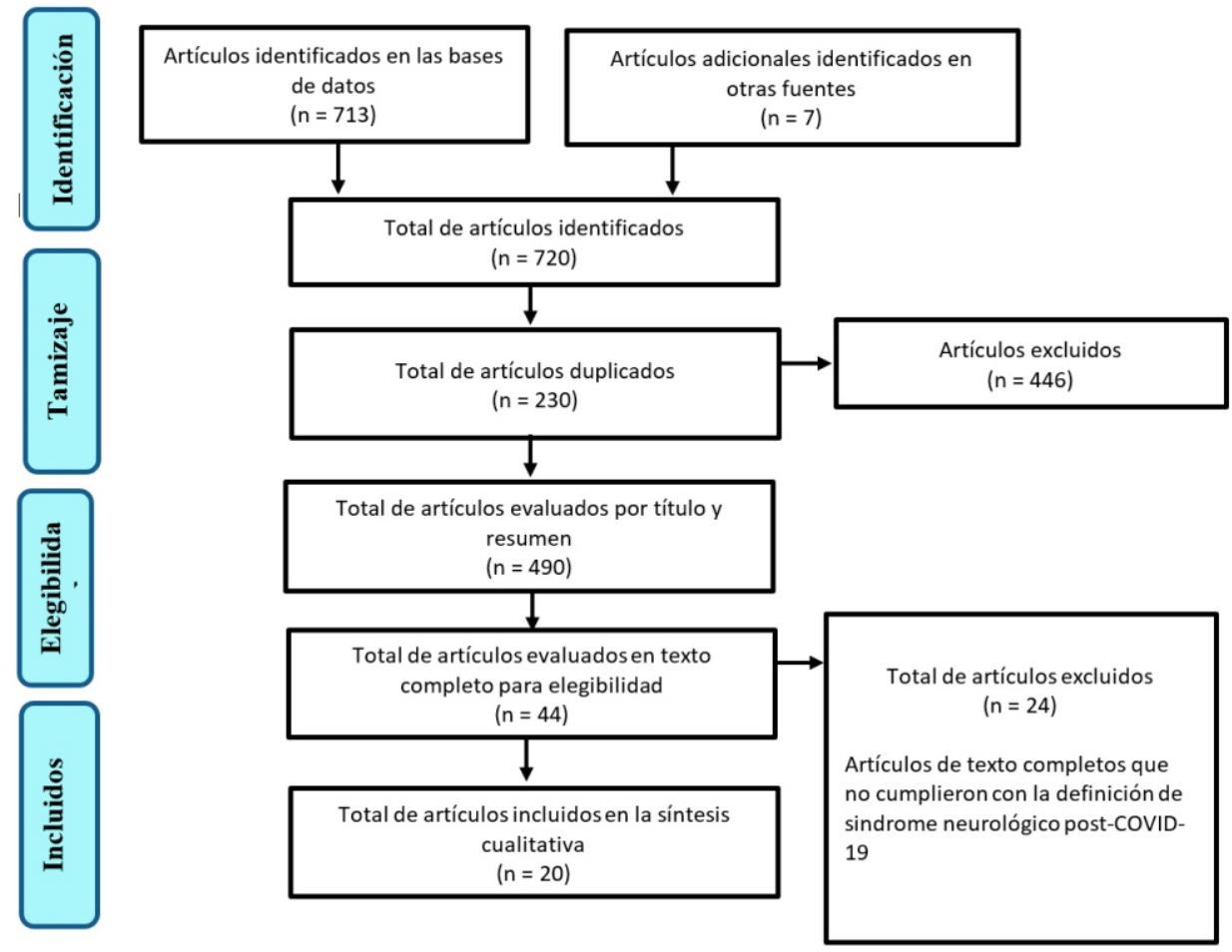

Figura 1.

Diagrama de flujo de la selección de los estudios. 
Revista del Cuerpo Médico del HNAAA, Vol 14 (3) - 2021

Fisiopatología de la infección (SARS-CoV-2 y receptores ECA-2)

La fase inicial para el desarrollo de la infección es la inhalación de partículas virales, presentes en gotitas respiratorias de una persona infectada. El virión ingresa en la cavidad nasal de una persona sana y se une a células caliciformes y ciliadas de la nariz que exponen receptores de enzima convertidora de angiotensina 2 (ECA2). Producto de ello, podría desencadenarse una respuesta innata circunscrita, y posteriormente, la replicación del virus con el consiguiente descenso hacia el tracto respiratorio. En tanto que los viriones se multiplican y se extienden, suele desencadenarse una firme respuesta innata a través de la localización de los viriones por los receptores de reconocimiento de patrones (RRP). Además, esto conduce en fases iniciales a la expresión del interferón tipo 1, lo que promueve en las células una actividad antivírica a través de quimiocinas y citocinas inflamatorias ${ }^{(4)}$.

Los viriones se desplazan hacia el tracto respiratorio inferior y logran alcanzar los alvéolos, en donde se vinculan con los neumocitos tipo 2 e inician la replicación. Conforme se produzca la apoptosis de los neumocitos, se liberan mediadores inflamatorios, encargados de atraer neutrófilos y macrófagos. Estos macrófagos a su vez, liberan citoquinas y factor de necrosis tumoral a (FNT-a). Las diversas citoquinas desencadenan un ataque, incitando la liberación de quimiocinas, factor de crecimiento endotelial vascular (VEGF) y disminución de E- cadherina. Esto provoca vasodilatación e incremento de permeabilidad capilar, y consecuentemente, aumento de edema intersticial y alveolar. La estimulación de los neutrófilos producirá especies reactivas de oxígeno (ROS) y proteasas que van a contribuir a la destrucción de los neumocitos tipo 1 y 2 , lo cual conlleva a una disminución del intercambio gaseoso y subsecuentemente a un colapso alveolar. Asimismo, los neumocitos muertos, quedan en los alvéolos, saturándose de líquido, restos celulares, neutrófilos y macrófagos, induciendo una consolidación pulmonar ${ }^{(5)}$.

\section{Interacción del virus y el sistema nervioso}

Se ha visto que el SARS-CoV-2, tiene un nivel de afectación multisistémico, incluyendo los sistemas respiratorio, nervioso, cardiovascular, musculo esquelético y gastrointestinal, por ello, la sintomatología varía según cada paciente. A pesar de que no está del todo claro cuál es la vía de acceso del virus al sistema nervioso central (SNC), se ha propuesto la hipótesis de que el SARS-CoV-2 puede ingresar mediante la difusión hematógena o neural, siendo viable una diseminación nerviosa por la polarización de las neuronas. Incluso también, por medio del epitelio olfatorio a lo largo de los axones de las neuronas sensoriales olfativas mediante un transporte retrógrado. Por otro lado, existe certeza de la presencia de material genético y proteínas del SARS-CoV-2 en tejidos del sistema nervioso, por lo que nos indica que el virus consigue penetrar y dañar el sistema nervioso ${ }^{(6)}$.

Otra probabilidad es que la proteína de pico o S del SARS-CoV2 posee una elevada atracción por el receptor de la ECA2. Estos receptores se manifiestan incontablemente en el endotelio capilar de diversas partes del cuerpo, incluido el cerebro, en el cual, el virus puede ingresar por medio de la barrera hematoencefálica, lesionar el sistema nervioso e inducir enfermedades neurológicas. Así mismo, se ha indicado que las células gliales y las neuronas exponen receptores ECA2, lo que las transforma en un objetivo potente del SARS-CoV-2. Además, teniendo en cuenta el efecto pro inflamatorio de la ECA2, es factible que su intervención en el cerebro a lo largo de la infección por COVID -19 , conlleve a una alteración en la autorregulación de la presión arterial, lo que produciría la interrupción de la pared arterial ${ }^{(7)}$. Sin embargo, en el estudio realizado por Yang $L$ et al. ${ }^{(8)}$, los niveles de expresión de ECA2 no parecen relacionarse con el potencial de infectividad del virus, ya que se observó el acceso de este a neuronas dopaminérgicas derivadas de células madre pluripotenciales a pesar de los bajos niveles de ECA2.

El anterior hallazgo planteó la búsqueda de otros factores que puedan estar implicados en los mecanismos que usa el virus para ingresar a las células. En esa línea, se halló que la proteína de pico del virus contiene un sitio de escisión para la proteasa furina, ausente en él, y es aquí donde se menciona el rol de la neuropilina-1 (NRP1), que es un receptor que se expresa abundantemente en el epitelio respiratorio y olfatorio (en este último también se ha detectado NRP1 en progenitores neuronales olfatorios). Ya que es conocido que la NRP1 se une a sustratos escindidos con furina, se cree que potencia la característica infectiva del virus. Otro receptor estudiado, debido al papel que desempeñaba en el SARS-CoV, es el CD147 (conocido como basigin), y se encontró que, al igual que con el SARS-CoV, mediaba la invasión viral cuando se unía a la proteína de pico ${ }^{(9)}$.

Por tanto, esta entrada por medio de los diversos mecanismos mencionados y la consiguiente replicación del virus, es sugerido como el causante de la desviación de la capacidad bioenergética de las células infectadas y es señalado como un factor crucial que afecta funciones neurológicas integradoras que se encargan de regular procesos cognitivos y afectivos importantes ${ }^{(10)}$.

\section{Síndrome neurológico post-Covid-19}

Nuevo síndrome caracterizado por manifestaciones neurológicas y neuropsiquiátricas persistentes por un periodo de tiempo de al menos 3 meses en pacientes después de la resolución de la sintomatología respiratoria provocada por la Covid-19. Este síndrome se manifiesta por las secuelas que deja el daño directo o indirecto del virus, por medio de mecanismos propios del sistema inmune, sobre el sistema nervioso central durante la fase aguda o incluso en un periodo post agudo por factores inmunológicos, esto implica un origen multifactorial y le da un carácter heterogéneo ${ }^{(11)}$.

Se han revisado múltiples estudios que muestran como resultado o parte de sus resultados las secuelas neurológicas que pueden manifestarse luego de un período de más de 3 meses desde la infección aguda por la Covid-19. Estos estudios, realizados en múltiples países, nos muestran el tipo de estudio, el tamaño de la muestra, el método por el cual los pacientes fueron diagnosticados por COVID-19, las manifestaciones neurológicas o psiquiátricas y el tiempo en las que éstas se presentaron (Tabla 1). La mayoría de estudios que hemos incluido en esta revisión tienen en común que la prueba diagnóstica fue la reacción en cadena de la polimerasa con transcriptasa inversa (RT-PCR), la cual es una prueba variante del PCR que ha sido de gran utilidad en esta pandemia. 
Tabla 1. Características de los estudios incluidos.

\begin{tabular}{|c|c|c|c|c|c|c|}
\hline Autor, año & País & Tipo de estudio & $\begin{array}{l}\text { Tamaño de } \\
\text { muestra }\end{array}$ & $\begin{array}{l}\text { Método de } \\
\text { diagnóstico }\end{array}$ & Clínica neurológica más frecuente & Tiempo \\
\hline Soares FHC et al (2021) & Brasil & Transversal & 46 & RT-PCR IgM & $\begin{array}{l}\text { Fatiga }(68,8 \%) \text {, hipogeusia }(50,3 \%) \text {, cefalea }(39,1 \%) \text {, } \\
\text { anosmia }(39,1 \%) \text {, }\end{array}$ & 3 meses \\
\hline Lu Y et al (2020) & China & Cohorte & 60 & RT-PCR & Cambio de humor $(16,67 \%)$, fatiga $(6,67 \%)$, & 3 meses \\
\hline Xiong Q et al (2021) & China & Cohorte & 538 & RT-PCR & Síntomas psicosociales $(22,7 \%)$, fatiga $(28,3 \%)$, & 3 meses \\
\hline Huang $\mathrm{Ch}$, et al (2021) & China & Cohorte & 1733 & Anticuerpos & Ansiedad o depresión (23\%), & 6 meses \\
\hline Tawfik HM et al (2021) & Egipto & Cohorte & 120 & RT-PCR & Anosmia $(41,7 \%)$, fatiga $(37,5 \%)$, cefalea $(28,3 \%)$, & 3 meses \\
\hline $\begin{array}{l}\text { Fernández-de-las-Peñas, C et } \\
\text { al (2021) }\end{array}$ & España & Casos y controles & 183 & RT-PCR & $\begin{array}{l}\text { Fatiga }(61,5 \%) \text {, síntomas depresivos }(22,1 \%) \text {, pérdida de } \\
\text { memoria }(16,4 \%) \text {, síntomas de ansiedad }(11,5 \%) \text {, pérdida } \\
\text { de concentración }(11,4 \%) \text {, ageusia/hipogeusia }(2,5 \%) \text {, } \\
\text { anosmia/hiposmia }(1,6 \%) \text {, }\end{array}$ & 7 meses \\
\hline Petracek LS et al (2021) & $\begin{array}{l}\text { Estados Unidos de } \\
\text { América }\end{array}$ & Reporte de caso & 3 & RT-PCR & Encefalomielitis Miálgica/Síndrome de Fatiga Crónica & 6 meses \\
\hline Taquet $M$, et al (2021)l & $\begin{array}{l}\text { Estados Unidos de } \\
\text { América }\end{array}$ & Cohorte & 236379 & RT-PCR & $\begin{array}{l}\text { Trastorno de ansiedad }(17,4 \%) \text {, depresión }(13,7 \%) \text {, } \\
\text { insomnio }(5,4 \%) \text {, ictus isquémico }(2,1 \%) \text {, psicosis }(1,4 \%) \text {, } \\
\text { demencia }(0,7 \%) \text {, parkinsonismo }(0,1 \%) \text {, }\end{array}$ & 6 meses \\
\hline Logue Jk et al (2021) & $\begin{array}{l}\text { Estados Unidos de } \\
\text { América }\end{array}$ & Cohorte & 177 & RT-PCR & $\begin{array}{l}\text { Fatiga }(13,6 \%) \text {, anosmia o ageusia }(13,6 \%) \text {, confusión } \\
\text { mental }(2,3 \%) \text {, }\end{array}$ & 6 meses \\
\hline Garrigues E et al (2020) & Francia & Transversal & 120 & $\begin{array}{l}\text { RT-PCR TAC } \\
\text { tórax }\end{array}$ & $\begin{array}{l}\text { Fatiga }(55 \%) \text {, disnea }(42 \%) \text {, pérdida de memoria }(34 \%) \text {, } \\
\text { trastornos de concentración y sueño ( } 28 \% \text { y } 30,8 \%) \text {, }\end{array}$ & 110 días \\
\hline Dennis A et al (2021) & Inglaterra & Cohorte & 201 & RT-PCR & Fatiga $(98 \%)$, cefalea $(83 \%)$, & 4 meses \\
\hline Mazza MG et al (2021) & Italia & Cohorte & 226 & RT-PCR & $\begin{array}{l}\text { Trastorno depresivo mayor }(8,85 \%) \text {, Trastorno de } \\
\text { Ansiedad }(8,85 \%) \text {, insomnio }(3,1 \%) \text {, }\end{array}$ & 3 meses \\
\hline Nuzzo, D et al (2021) & Italia & Reporte de caso & 1 & RT-PCR & Depresión & 9 meses \\
\hline Venturelli S et al (2021) & Italia & Cohorte & 767 & RT-PCR & Fatiga $(51 \%)$, estrés postraumático $(30,5 \%)$, & 81 días \\
\hline Stavem K et al (2021) & Noruega & Cohorte & 938 & RT-PCR & Anosmia (12\%), disgeusia (10\%), cefalea $(6 \%)$ & 6 meses \\
\hline Stavem K et al (2021) & Noruega & Cohorte & 938 & RT-PCR & Fatiga (46\%), & 6 meses \\
\hline Goërtz YMJ et al (2020) & Países Bajos y Bélgica & Cohorte & 2213 & RT-PCR & Fatiga $(87 \%)$, cefalea $(38 \%)$, anosmia $(13 \%)$, ageusia $(11 \%)$ & 79 + 17 días \\
\hline Davis HE, et al, (2020) & Multicéntrico & Cohorte & 3762 & $\begin{array}{c}\text { RT-PCR } \\
\text { Anticuerpos }\end{array}$ & $\begin{array}{l}\text { Fatiga }(77,7 \% \text { a } 80,3 \%) \text {, disfunción cognitiva }(55,34 \text { a } \\
58,8 \%) \text {, }\end{array}$ & 7 meses \\
\hline
\end{tabular}

La fatiga es uno de los síntomas más frecuentes hallados en múltiples estudios ${ }^{(12)}$, según Goërtz et al. ${ }^{(13)}$, en un estudio de cohortes, en la cual se evaluó un total de 2113 pacientes, basándose en comorbilidades, estado de salud, inicio de síntomas, diagnóstico de COVID-19 y presencia de 29 síntomas en el momento de la aparición y durante el seguimiento, observándose que en pacientes hospitalizados y no hospitalizados con COVID-19, se presentaron diversos síntomas aproximadamente 3 meses después del inicio de los síntomas, siendo la fatiga, el más prevalente. Stavem et al. ${ }^{(14)}$ también describieron que en pacientes no hospitalizados con una PCR positiva para SARS-CoV-2, el $46 \%$ de los participantes indicaron fatiga aproximadamente 4 meses después del inicio de los síntomas, teniendo asociación con el sexo femenino, así como indicios de una depresión previa. De hecho, un estudio de cohorte realizado por Venturelli et al. ${ }^{(15)}$ halló que los pacientes que accedieron a un programa de seguimiento multidisciplinario en una mediana de 81 días luego del alta médica, el $51,4 \%$ todavía mostraba síntomas, refiriendo frecuentemente fatiga, y el $30,5 \%$ aún presentaba consecuencias psicológicas. Otra alteración mencionada con cierta frecuencia ha sido la encefalomielitis/síndrome de fatiga crónica (ME/CFS), ya que según el estudio de Petracek et al. ${ }^{(16)}$, la COVID-19 puede desencadenar ME/CFS en 
adolescentes y adultos jóvenes.

La cefalea se ha destacado por ser un síntoma relevante y frecuente durante la fase aguda de la infección, sin embargo, puede prolongarse incluso en el periodo después de la recuperación, llegando a cumplir criterios de dolor de cabeza persistente diario de la Clasificación Internacional de Trastornos del Dolor de Cabeza. Los mecanismos implicados que permitan esta persistencia de la cefalea no están establecidos aún y en los estudios de imagen a los 2 meses pueden observarse que las estructuras nerviosas y sanguíneas no presentan alteración alguna ${ }^{(17)}$. Diversos estudios ${ }^{(11,18,19,20)}$ reportan cefalea persistente por más de 3 meses, pudiendo llegar hasta un $83 \%$ en pacientes que estuvieron hospitalizados por COVID-19.

Dentro de los hallazgos de múltiples estudios se ha indicado una serie de manifestaciones psiquiátricas, probablemente explicado por el impacto de la neuroinflamación que se produce durante la infección y es determinante en el inicio o progresión de trastornos neuropsiquiátricos. Las alteraciones más frecuentes son: trastornos de ansiedad y depresión, alteraciones del sueño y estrés postraumático ${ }^{(21)}$.

El hallazgo de estrés postraumático ha sido relevante en algunos estudios, como el descrito por Venturelli et al. ${ }^{(15)}$, donde se halló hasta un 30,5\% de síntomas postraumáticos; sin embargo, en un estudio de Simani et al. ${ }^{(22)}$, la tasa de prevalencia de estrés postraumático llega solo a un $5,8 \%$; esta alteración puede estar en relación a las altas tasas de hospitalización y de estancia hospitalaria prolongada debido a la magnitud con la que se desarrolló la enfermedad en ellos. Los reportes de depresión, ansiedad y/o trastornos del sueño revelan la importancia de realizar un seguimiento a los pacientes luego del alta hospitalaria, ya que factores como las medidas de aislamiento aplicadas por los gobiernos y la incertidumbre pueden agravar o ser parte desencadenante de estos trastornos ${ }^{(10,23-27)}$. Huang et al. ${ }^{(28)}$, en un estudio de cohorte ambidireccional de pacientes con COVID-19 que habían sido dados de alta, se les entrevistó con diversos cuestionarios para evaluar los síntomas. Los sobrevivientes, con una mediana de tiempo de seguimiento de 186 días luego de la aparición de los síntomas, presentaron ansiedad o depresión en un $23 \%$, siendo común en mujeres. Además de estos síntomas, en el estudio unicéntrico de Garrigues et al. ${ }^{(29)}$, con más de 100 días posteriores al alta, se detectaron síntomas a través de un cuestionario telefónico, donde los datos relevantes fueron pérdida de memoria (34\%) y trastornos de la concentración y del sueño (28\% y $30,8 \%$, correspondientemente).

Otro hallazgo notable, luego de 3 meses de sufrir una infección aguda por la COVID-19, fue la persistencia de anosmia en algunos pacientes, llegando a valores como $41,7 \%$ o $39,1 \%$ en dos estudios $(18,19)$. Sin embargo, esta alteración junto a la ageusia, ha sido reportada con un importante descenso a los 6 meses, en valores que apenas llegan al 13\% en otros estudios revisados ${ }^{(6,7,14,16,17)}$.

\section{CONCLUSIONES}

Según lo referido, existe evidencia que soporta la presencia de nuevas enfermedades secundarias a la infección índice por el SARS-CoV-2; los investigadores la han denominado síndrome neurológico post-Covid-19 y representa la presencia de manifestaciones neurológicas y psiquiátricas de más de tres meses de duración. Esta realidad abre una línea de investigación para seguir ampliando los conocimientos de esta nueva entidad; por otro lado, advertir a la comunidad médica para una mayor vigilancia y detección en la atención primaria de estas manifestaciones, que requieren un diagnóstico y tratamiento oportuno.

\section{REFERENCIAS BIBLIOGRÁFICAS}

1. Wang C, Horby PW, Hayden FG, Gao GF. A novel coronavirus outbreak of global health concern. The Lancet. 2020 February 15 [citado 02

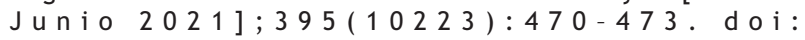
https://doi.org/10.1016/S0140-6736(20)30185-9.

2. Amanat F, Krammer F. SARS-CoV-2 Vaccines: Status Report. Immunity. 2020 April 14 [citado 02 Junio 2021];52(4):583-589. doi: https://doi.org/10.1016/j.immuni.2020.03.007.

3. Wijeratne T, Crewther S. Post-COVID 19 Neurological Syndrome (PCNS); a novel syndrome with challenges for the global neurology community. J Neurol Sci. 2020 December 15 [citado 02 Junio 2021];419:117179. doi: 10.1016/j.jns.2020.117179.

4. Imai Y, Kuba K, Ohto-Nakanishi T, Penninger JM. Angiotensinconverting enzyme 2 (ACE2) in disease pathogenesis. Circ J. 2010 March [citado 02 Junio 2021];74(3):405-410. doi: 10.1253/circj.cj10-0045.

5. Singh SP, Pritam M, Pandey B, Yadav TP. Microstructure, pathophysiology, and potential therapeutics of COVID-19: A comprehensive review. J Med Virol. 2021 January [citado 02 Junio 2021];93(1):275-299. doi: 10.1002/jmv.26254.

6. Tang SW, Helmeste D, Leonard B. Inflammatory neuropsychiatric disorders and COVID-19 neuroinflammation. Acta Neuropsychiatr. 2021 April 30 [citado 03 Junio 2021]:1-13. doi: 10.1017/neu.2021.13.

7. Stefano GB, Büttiker P, Weissenberger S, Martin A, Ptacek R, Kream RM. Editorial: The Pathogenesis of Long-Term Neuropsychiatric COVID-19 and the Role of Microglia, Mitochondria, and Persistent Neuroinflammation: A Hypothesis. Med Sci Monit [Internet]. 10 de mayo de 2021 [citado 2 Junio 2021];27. doi: 10.12659/MSM.933015.

8. Yang L., Han Y., Nilsson-Payant B.E., Gupta V., Wang P., Duan X., Tang X., Zhu J., Zhao Z., Jaffre F. A Human Pluripotent Stem Cell-based Platform to Study SARS-CoV-2 Tropism and Model Virus Infection in Human Cells and Organoids. Cell Stem Cell. 2020 [citado 03 Junio 2021];27:125-136. doi: 10.1016/j.stem.2020.06.015.

9. Cantuti-Castelvetri L, Ojha R, Pedro LD, Djannatian M, Franz J, Kuivanen $S$, et al. Neuropilin-1 facilitates SARS-CoV-2 cell entry and infectivity. Science. 2020 November 13 [citado 03 Junio 2021]; 370(6518):856-860. doi: 10.1126/science. abd2985.

10. Nuzzo D, Cambula G, Bacile I, Rizzo M, Galia M, Mangiapane P, et al. Long-Term Brain Disorders in Post Covid-19 Neurological Syndrome (PCNS) Patient. Brain Sci [Internet]. 2021 April 2 [citado 03 Junio 2021];11(4):454. doi: 10.3390/brainsci11040454.

11. Davis HE, Assaf GS, McCorkell L, Wei H, Low RJ, Re'em Y, et al. Characterizing Long COVID in an International Cohort: 7 Months of Symptoms and Their Impact. medRxiv. 2021 December 27 [citado 15 Jun i o 2021 ]; 2020.12 .24 .20248802 . doi : https://doi.org/10.1101/2020.12.24.20248802.

12. Logue JK, Franko NM, McCulloch DJ, McDonald D, Magedson A, Wolf $\mathrm{CR}$, et al. Sequelae in Adults at 6 Months After COVID-19 Infection. JAMA Netw Open. 2021 February 19 [citado 15 Junio 2021];4(2):e210830. doi: 10.1001/jamanetworkopen.2021.0830.

13. Goërtz YMJ, Van Herck M, Delbressine JM, Vaes AW, Meys R, Machado FVC, et al. Persistent symptoms 3 months after a SARS-CoV-2 infection: the post-COVID-19 syndrome? ERJ Open Res. octubre de 2020 [citado 24 Junio 2021];6(4). doi: 10.1183/23120541.005422020.

14. Stavem K, Ghanima W, Olsen MK, Gilboe HM, Einvik G. Prevalence and determinants of fatigue after covid-19 in non-hospitalized subjects: A population-based study. International Journal of Environmental Research and Public Health. 2021 [citado 24 Junio 2021];18(4):1-11. doi: 10.3390/ijerph18042030.

15. Venturelli S, Benatti SV, Casati M, Binda F, Zuglian G, Imeri G, et al. Surviving COVID-19 in Bergamo province: a post-acute outpatient re- 
evaluation. Epidemiology \& Infection [Internet]. 2021 January 19 [citado 24 de junio de 2021];149. doi: 10.1017/S0950268821000145.

16. Petracek LS, Suskauer SJ, Vickers RF, Patel NR, Violand RL, Swope RL, et al. Adolescent and Young Adult ME/CFS After Confirmed or Probable COVID-19. Front Med. 2021 April 29 [citado 20 Junio 2021];8:668944. doi: $10.3389 /$ fmed.2021.668944.

17. Liu JWTW, de Luca RD, Mello Neto HO, Barcellos I. Post-COVID-19 Syndrome? New daily persistent headache in the aftermath of COVID19. Arq Neuropsiquiatr. 2020 November [citado 24 Junio 2021];78(11):753-4. doi: https://doi.org/10.1590/0004 $282 \times 20200187$

18. Soares FHC, Kubota GT, Fernandes AM, Hojo B, Couras C, Costa BV, et al. Prevalence and characteristics of new-onset pain in COVID-19 survivours, a controlled study. Eur J Pain. 2021 April 8 [citado 27 Junio 2021]:ejp.1755. doi: 10.1002/ejp.1755.

19. Tawfik HM, Shaaban HM, Tawfik AM. Post-covid-19 syndrome in egyptian healthcare staff: Highlighting the carers sufferings. Electronic Journal of General Medicine [Internet]. 2021 [citado 27 Junio 2021];18(3). doi: https://doi.org/10.29333/ejgm/10838.

20. Dennis A, Wamil M, Alberts J, Oben J, Cuthbertson DJ, Wootton D, et al. Multiorgan impairment in low-risk individuals with post-COVID-19 syndrome: A prospective, community-based study. BMJ Open [Internet]. 2021 [citado 27 Junio 2021];11(3). doi: 10.1136/bmjopen2020-048391.

21. Stavem K, Ghanima W, Olsen MK, Gilboe HM, Einvik G. Persistent symptoms 1.5-6 months after COVID-19 in non-hospitalised subjects: A population-based cohort study. Thorax. 2021 [citado 27 Junio 2021];76(4):405-7. doi: 10.1136/thoraxjnl-2020-216377.

22. Simani L, Ramezani M, Darazam IA, Sagharichi M, Aalipour MA, Ghorbani F, et al. Prevalence and correlates of chronic fatigue syndrome and post-traumatic stress disorder after the outbreak of the COVID-19. Journal of NeuroVirology. 2021 [citado 27 Junio 2021]; 27(1):154-9. doi: 10.1007/s13365-021-00949-1.

23. Fernández-de-Las-Peñas C, Torres-Macho J, Velasco-Arribas M, AriasNavalón JA, Guijarro C, Hernández-Barrera V, et al. Similar prevalence of long-term post-COVID symptoms in patients with asthma: A case-control study. J Infect. 2021 May 3 [citado 27 Junio 2021]; S0163-4453(21)00222-X. doi: 10.1016/j.jinf.2021.04.034.

24. Romero-Duarte Á, Rivera-Izquierdo M, Guerrero-Fernández de Alba I, Pérez-Contreras M, Fernández-Martínez NF, Ruiz-Montero R, et al. Sequelae, persistent symptomatology and outcomes after COVID-19 hospitalization: the ANCOHVID multicentre 6-month follow-up study. BMC Medicine. 2021 May 20 [citado 28 Junio 2021];19(1):129. doi: 10.1186/s12916-021-02003-7.

25. Taquet M, Geddes JR, Husain M, Luciano S, Harrison PJ. 6-month neurological and psychiatric outcomes in 236379 survivors of COVID19: a retrospective cohort study using electronic health records. Lancet Psychiatry. 2021 May [citado 28 Junio 2021];8(5):416-27. doi: 10.1016/S2215-0366(21)00084-5.

26. Mazza MG, Palladini M, De Lorenzo R, Magnaghi C, Poletti S, Furlan R, et al. Persistent psychopathology and neurocognitive impairment in COVID-19 survivors: Effect of inflammatory biomarkers at threemonth follow-up. Brain, Behavior, and Immunity. 2021 May [citado 28 Junio 2021];94:138-47. doi: 10.1016/j.bbi.2021.02.021.

27. Xiong Q, Xu M, Li J, Liu Y, Zhang J, Xu Y, et al. Clinical sequelae of COVID-19 survivors in Wuhan, China: a single-centre longitudinal study. Clin Microbiol Infect. 2021 January [citado 28 Junio 2021];27(1):89-95. doi: 10.1016/j.cmi.2020.09.023.

28. Huang $C$, Huang $L$, Wang $Y, L i X$, Ren $L$, Gu X, et al. 6-month consequences of COVID-19 in patients discharged from hospital: a cohort study. Lancet. 2021 [citado 28 Junio 2021];397(10270):22032. doi: 10.1016/S0140-6736(20)32656-8.

29. Garrigues E, Janvier P, Kherabi Y, Le Bot A, Hamon A, Gouze H, et al. Post-discharge persistent symptoms and health-related quality of life after hospitalization for COVID-19. J Infect. 2020 December [citado 28 Junio 2021];81(6):e4-6. doi: 10.1016/j.jinf.2020.08.029.

30. Lu Y, Li X, Geng D, Mei N, Wu P-Y, Huang C-C, et al. Cerebral MicroStructural Changes in COVID-19 Patients - An MRI-based 3-month Follow-up Study. EClinicalMedicine. 2020 August [citado 28 Junio 2021];25:100484. doi: 10.1016/j.eclinm.2020.100484. 